\title{
Padrões de infestação de Comunidades de Plantas Daninhas no Agroecossistema de Cana-Crua ${ }^{1}$
}

\author{
Weed Plant Infestation Patterns in Raw Sugarcane Agricultural Systems
}

\begin{abstract}
KUVA, M.A. ${ }^{2}$, FERRAUDO, A.S. ${ }^{3}$, PITELLI, R.A. ${ }^{4}$, ALVES, P.L.C.A. ${ }^{5}$ e SALGADO, T.P. ${ }^{2}$
RESUMO - Objetivou-se neste trabalho a obtenção de padrões de infestação de plantas daninhas na cultura de cana-de-açúcar com histórico de colheita mecanizada sem queima prévia da palha. Foram realizadas amostragens em 28 talhões na região de Ribeirão Preto, SP; em cada talhão foram demarcadas unidades de avaliação e coleta, na proporção de duas por hectare, que consistiram de áreas (quatro linhas de 4 metros de comprimento) mantidas sem controle de plantas daninhas e onde foram realizadas as amostragens de plantas emergidas. As amostragens foram realizadas aos 120 dias após o corte, com quadrados vazados $(0,5 \times 0,5 \mathrm{~m})$ lançados aleatoriamente duas vezes em cada uma das unidades de avaliação e coleta. Com os dados obtidos, calculou-se a importância relativa e o índice de agregação das espécies ou grupo de espécies. Esses indices foram usados no processamento da análise de agrupamento hierárquica, utilizando como medida de semelhança a distância euclidiana e como estratégia de agrupamento o método UPGMA (Unweighted Pair-Group Method using arithmetic Averages). Foi possivel distinguir quatro grupos em função da importância relativa e cinco grupos de talhões em função do índice de agregação; dentro de alguns grupos houve formação de subgrupos.
\end{abstract}

Palavras-chave: análise de agrupamento, palha, fitossociologia, índice de agregação, Saccharum spp.

\begin{abstract}
The aim of this work was to obtain the weed infestation patterns in sugarcane plantation with a history of mechanical harvest without previous straw burning. Twenty-eight plots were chosen in the Ribeirão Preto region, in the state of São Paulo, with evaluation and collection units being demarcated in each plot, in a proportion of two per hectare, which consisted of areas (4 rows of 4 meters in length) maintained free of any weed control method. Samplings were carried out at 120 days after cut with quadrants $(0.5 \mathrm{~m} \times 0.5 \mathrm{~m})$ cast twice at random at each evaluation and collection unit. The following phytosociological indices were calculated from the obtained data: relative importance and aggregation index of species or group of species. Grouping analysis was carried out utilizing as the similarity coefficient the Euclidian distance and the Unweighted Pair-Group Method using arithmetic averages. It was possible to distinguish four groups as a function of relative importance and five plot groups as a function of the aggregation index, with formation of subgroups within some groups.
\end{abstract}

Keywords: cluster analysis, straw, phytosociology, aggregation index, Saccharum spp.

1 Recebido para publicação em 24.1.2008 e na forma revisada em 23.7.2008.

Parte da tese do primeiro autor apresentada à Unesp - Jaboticabal para obtenção do título de Doutor em Agronomia (Produção Vegetal).

2 Herbae Consultoria e Projetos Agrícolas Ltda., Rua José Antonio Fernandes Sobrinho, 118, B. Aparecida, 14882-046, JaboticabalSP, <mkuva@herbae.com.br>; ${ }^{3}$ Prof. Assistente, Dr., Dep. de Ciências Exatas da Unesp - Jaboticabal. Via de acesso Prof. Paulo Donato Castellane, km 5, s/n, 14870-000, Jaboticabal-SP, ${ }^{4}$ Prof. Titular, Dep. de Biologia Aplicada à Agropecuária da Unesp Jaboticabal-SP; ${ }^{5}$ Prof. Assistente, Dr., Dep. de Biologia Aplicada à Agropecuária da Unesp - Jaboticabal-SP. 


\section{INTRODUÇÃO}

Em se tratando de fatores bióticos, as plantas daninhas estão entre os principais componentes do agroecossistema que interferem no desenvolvimento e na produtividade das culturas. A presença dessas plantas pode interferir no processo produtivo, competindo pelos recursos do meio, hospedando pragas e doenças ou interferindo nas práticas culturais e na colheita (Pitelli, 1985). Os recursos despendidos no controle das plantas daninhas correspondem, em média, a $8 \%$ do custo total de produção da cana-planta e de 7 a $14 \%$ da cana-soca (FNP, 2006).

A utilização de herbicidas é o método de controle mais empregado, pois as áreas que a cana-de-açúcar ocupa são bastante extensas. Para melhor utilização dessa tecnologia, o conhecimento das caracteristicas dos herbicidas, das condições do ambiente e da composição específica da comunidade infestante são fatores relevantes. O conhecimento mais aprofundado da composição da flora de planta daninha poderá contribuir, sem dúvida, na redução das despesas destinadas ao controle de plantas daninhas, pois os recursos poderão ser empregados de maneira mais criteriosa e específica, em função das características da área, dos herbicidas e da comunidade infestante.

Alguns trabalhos têm se dedicado a descrever os agroecossistemas quanto à composição específica de plantas daninhas e os reflexos das práticas culturais nessa composição. Em grande parte deles tem se recorrido aos indices fitossociológicos (Lara et al., 2003; Souza et al., 2003; Kuva et al., 2007), e a comparação entre áreas, na maioria dos casos, tem sido realizada pelo indice de similaridade descrito em Odum (1985). Esse coeficiente apresenta como limitação o fato de considerar somente a composição de espécies, deixando de lado o tamanho das populações, o acúmulo de biomassa e suas distribuições. Além disso, com essa técnica somente é possivel comparar as áreas duas a duas (Macedo et al., 2003), o que dificulta a obtenção de padrões de infestação. O agrupamento de talhões com algumas similaridades, se realizado com ferramentas estatísticas e se considerados os indices relevantes e práticos, poderá ser bastante útil na tomada de decisões para controle de plantas daninhas.
Um procedimento estatístico que pode ser utilizado para o estabelecimento de grupos padrões é a análise multivariada de dados pela técnica de agrupamento, que permite a construção de dendrogramas, que arranja os grupos em função do coeficiente de dissimilaridade, de distância euclidiana ou de outros coeficientes. Na ciência das plantas daninhas, Rowe et al. (1997) e Winkler et al. (2003) recorreram a esse tipo de análise de dados para estudar a divergência genética de Euphorbia esula e Euphorbia heterophylla, respectivamente.

Foi objetivo desta pesquisa verificar a possibilidade de obtenção de padrões de infestação de comunidades de plantas daninhas na cultura da cana-de-açúcar colhida mecanicamente e sem queima prévia da palha por meio de análise de agrupamento, utilizando como variáveis os indices fitossociológicos, importância relativa e indice de agregação das diferentes espécies e grupos de espécies.

\section{MATERIAL E MÉTODOS}

Os dados utilizados neste trabalho foram obtidos durante a safra de 2004, em 28 talhões de cana-soca com histórico de colheita mecanizada, localizados em usinas e fornecedores da região de Ribeirão Preto, SP. Esses talhões foram separados em três grupos, de acordo com a época de corte. O primeiro grupo foi composto por dez talhões colhidos no mês de julho (época 1 - soca-seca); o segundo, por dez talhões colhidos a partir da segunda quinzena de agosto até meados de setembro (época 2 - final da socaseca); e o terceiro, por oito talhões colhidos no final de safra, nos meses de outubro e novembro (época 3 - soca-úmida). Os talhões estudados, bem como as datas da realização das colheitas e as informações mais importantes, encontram-se na Tabela 1 . Os elementos meteorológicos registrados durante o periodo de coleta dos dados foram extraídos do acervo da Estação de Agrometeorologia do Departamento de Ciências Exatas e encontram-se sumarizadas na Figura 1.

Os talhões tiveram o tamanho limitado a cinco hectares e, após o corte da cana, seu perimetro foi georreferenciado (GPS Garmim 12; software GPS Track maker). Em cada ta1hão foram demarcadas áreas de coleta e avaliação, que ficaram sem aplicação de 
Tabela 1 - Localização e informações dos talhões utilizados para o levantamento e monitoramento de plantas daninhas

\begin{tabular}{|c|c|c|c|}
\hline Área & Fazenda - Município & Data de corte & Corte (crua) \\
\hline FSI-1 & $\begin{array}{l}\text { Faz. Santa Isabel - } \\
\text { Jaboticabal }\end{array}$ & $04 / 07 / 04$ & Quinto \\
\hline FBV-2 & $\begin{array}{c}\text { Faz. Bela Vista - } \\
\text { Jaboticabal }\end{array}$ & $16 / 07 / 04$ & Quinto \\
\hline NSF-3 & $\begin{array}{c}\text { Faz. N. Sra. de Fátima - } \\
\text { Taquaritinga }\end{array}$ & $02 / 07 / 04$ & Sexto \\
\hline FSC-4 & $\begin{array}{c}\text { Faz. Sta. Cecília - } \\
\text { Jaboticabal }\end{array}$ & $05 / 0704$ & Terceiro \\
\hline FBV-5 & $\begin{array}{c}\text { Faz. Bela Vista - } \\
\text { Jaboticabal }\end{array}$ & $19 / 07 / 04$ & Sexto \\
\hline UDP-6 & $\begin{array}{l}\text { Usina da Pedra - } \\
\text { Ribeirão Preto }\end{array}$ & $07 / 07 / 04$ & Quarto \\
\hline UAN-7 & $\begin{array}{l}\text { Faz. Trindade - } \\
\text { Pitangueiras }\end{array}$ & $30 / 07 / 04$ & Terceiro \\
\hline FSI-8 & $\begin{array}{c}\text { Faz. Sta. Isabel - } \\
\text { Jaboticabal }\end{array}$ & $27 / 07 / 04$ & Terceiro \\
\hline FSI-9 & $\begin{array}{l}\text { Faz. Sta. Isabel - } \\
\text { Jaboticabal }\end{array}$ & $25 / 07 / 04$ & Sétimo \\
\hline USB-10 & $\begin{array}{l}\text { Faz. Diamantina - } \\
\text { Taquaritinga }\end{array}$ & $14 / 05 / 04$ & Quinto \\
\hline USB-11* & $\begin{array}{c}\text { Faz. Cucuhy - } \\
\text { Guariba }\end{array}$ & $13 / 08 / 04$ & Terceiro \\
\hline COP-13 & $\begin{array}{l}\text { Faz. Coplana - } \\
\text { Guariba }\end{array}$ & $18 / 09 / 04$ & Segundo \\
\hline FM-14 & $\begin{array}{l}\text { Faz. Morumbi - } \\
\text { Guariba }\end{array}$ & $25 / 08 / 04$ & Terceiro \\
\hline FM-15 & $\begin{array}{l}\text { Faz. Morumbi - } \\
\text { Guariba }\end{array}$ & $28 / 08 / 04$ & Sexto \\
\hline FM -16 & $\begin{array}{c}\text { Faz. Morumbi - } \\
\text { Guariba }\end{array}$ & $05 / 09 / 04$ & Sexto \\
\hline FSA-17 & $\begin{array}{c}\text { Faz. Sto. Antônio - } \\
\text { Matão }\end{array}$ & $19 / 08 / 04$ & Terceiro \\
\hline FM -18 & $\begin{array}{l}\text { Faz. Morumbi - } \\
\text { Guariba }\end{array}$ & $12 / 09 / 04$ & Segundo \\
\hline USM-19 & $\begin{array}{l}\text { Faz. Porto Pinheiro } 17 \text { - } \\
\text { Pradópolis }\end{array}$ & $03 / 09 / 04$ & Quinto \\
\hline USM-20 & $\begin{array}{l}\text { Faz. Porto Pinheiro } 17 \text { - } \\
\text { Pradópolis }\end{array}$ & 03/09/04 & Quinto \\
\hline FSC-21 & $\begin{array}{c}\text { Faz. Sta. Cecília - } \\
\text { Jaboticabal }\end{array}$ & $12 / 09 / 04$ & Terceiro \\
\hline FBV-22 & $\begin{array}{c}\text { Faz. Bela Vista - } \\
\text { Jaboticabal } \\
\end{array}$ & $08 / 10 / 04$ & Segundo \\
\hline FBV-23 & $\begin{array}{c}\text { Faz. Bela Vista - } \\
\text { Jaboticabal } \\
\end{array}$ & $17 / 10 / 04$ & Quinto \\
\hline FBV-24 & $\begin{array}{c}\text { Faz. Bela Vista - } \\
\text { Jaboticabal }\end{array}$ & $17 / 10 / 04$ & Quarto \\
\hline FBV-25 & $\begin{array}{c}\text { Faz. Bela Vista - } \\
\text { Jaboticabal } \\
\end{array}$ & $21 / 10 / 04$ & Primeiro \\
\hline FSO-26 & $\begin{array}{c}\text { Faz. Santa Olívia - } \\
\text { Jaboticabal } \\
\end{array}$ & $06 / 11 / 04$ & Terceiro \\
\hline FSO-27 & $\begin{array}{c}\text { Faz. Santa Olívia - } \\
\text { Jaboticabal }\end{array}$ & $10 / 11 / 04$ & Sexto \\
\hline USM-28 & $\begin{array}{c}\text { Faz. Barrinha - } \\
\text { Barrinha } \\
\end{array}$ & $28 / 10 / 04$ & Quinto \\
\hline USM-29 & $\begin{array}{l}\text { Faz. Conceição - } \\
\text { Jaboticabal }\end{array}$ & $13 / 11 / 04$ & Terceiro \\
\hline
\end{tabular}

herbicidas e serviram de local para a realização dos levantamentos e da coleta das plantas daninhas. Essas áreas foram locadas aleatoriamente, na quantidade de duas a cada hectare, e constituídas de áreas retangulares (4 linhas $\mathrm{x} 4$ metros). A flora emergente foi avaliada

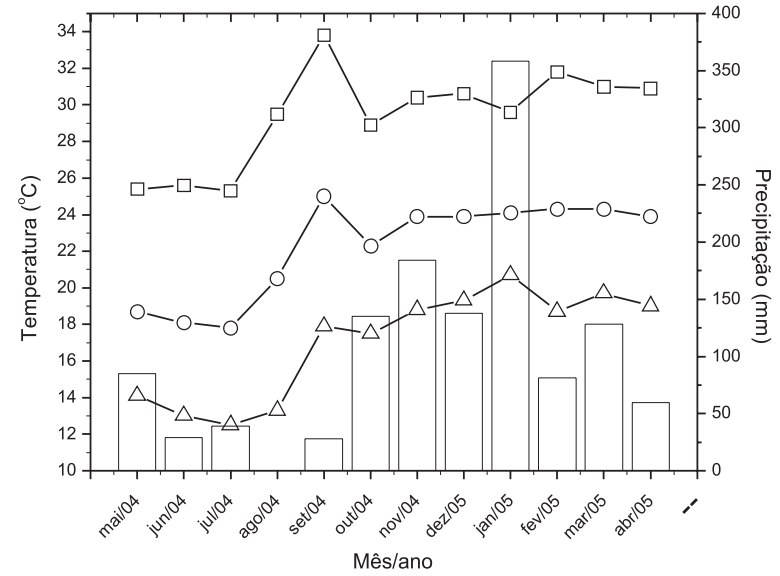

Fonte: Departamento de Ciências Exatas - Unesp Jaboticabal.

Figura 1 - Dados de precipitação (barras) e de temperaturas mínima $(\Delta)$, média $(\bigcirc)$ e máxima $(\square)$ registrados durante o período de coleta de dados.

aos 120 dias após o corte, utilizando-se de quadrados vazados de 0,5 x 0,5 m lançados ao acaso por duas vezes dentro de cada área de coleta e avaliação. As plantas abrangidas pelo quadrado de amostragem foram identificadas, contadas e recolhidas para determinação da massa seca acumulada por população, após secagem em estufa com aeração forçada e ajustada para $75{ }^{\circ} \mathrm{C}$, com posterior pesagem em balança com precisão de centigramas.

Os dados obtidos foram utilizados para obtenção da importância relativa (IR) e do índice de agregação $(\mathrm{V} / \mathrm{m})$, calculados segundo fórmulas contidas em Mueller-Dombois \& Ellenberg (1974). Esses indices foram calculados para a espécie Cyperus rotundus (CY) e para quatro conjuntos de espécies, obedecendo a critérios agronômicos: cordas-de-viola (CV), outras plantas de folha larga propagadas por sementes (FL), gramineas (GR) e perenes oriundas de rebrotas (PER). Os principais componentes de cada conjunto encontram-se na Tabela 2. A combinação dos grupos de plantas com os indices fitossociológicos originou dez variáveis: CY_IR; CY_V/m; CV_IR; CV_V/m; FL_IR; FL_V/m; GR_IR; GR_V/m; PER_IR; e PER_V/m. O estudo envolvendo as variáveis PER e GR ficou prejudicado pela baixa presença nos talhões, sendo estas analisadas individualmente. As demais - CY, CV e FL - foram submetidas à análise de agrupamento hierárquica (Hair et al., 2005), buscando organizar as 
Tabela 2 - Relação das principais espécies de plantas daninhas que compuseram os conjuntos estabelecidos para a análise de agrupamento dos talhões

\begin{tabular}{|c|l|}
\hline Conjunto & \multicolumn{1}{|c|}{ Componente } \\
\hline $\mathrm{CY}$ & Cyperus rotundus \\
\hline $\mathrm{CD}$ & Cynodon dactylon \\
\hline $\mathrm{CV}$ & $\begin{array}{l}\text { Ipomoea hederifolia, I. quamoclit, I. nil, I. } \\
\text { grandifolia, I. purpúrea, Merremia cissoides }\end{array}$ \\
\hline $\mathrm{FL}$ & $\begin{array}{l}\text { Amaranthus spp., Chamaesyce hissopifolia, Croton } \\
\text { glandulosus, Bidens pilosa, Solanum americanum, } \\
\text { Emilia sonchifolia, Tridax procumbens, Sida spp., } \\
\text { Conyza bonariensis, Physalis angulata, Commelina } \\
\text { benghalensis, Euphorbia heterophylla, Phylanthus } \\
\text { tenellus, } \text { Cleome affinis, Parthenium hysterophorus }\end{array}$ \\
\hline $\mathrm{GR}$ & $\begin{array}{l}\text { Brachiaria decumbens, Brachiaria plantaginea, } \\
\text { Eleusine indica, Panicum maximum, Digitaria spp., } \\
\text { Digitaria insularis, Cenchrus echinatus }\end{array}$ \\
\hline PER & $\begin{array}{l}\text { Vernonia polyanthes, Cissampelos glaberrima, }, \\
\text { Pyrostegia venusta, Talinum paniculatum, Mikania } \\
\text { cordifolia, além de outras espécies não identificadas }\end{array}$ \\
\hline
\end{tabular}

comunidades de plantas em grupos, segundo seus padrões de composição. Utilizou-se a distância euclidiana como medida de semelhança entre os talhões e, como estratégia de agrupamento, o método UPGMA: neste método, a distância entre dois grupos é definida como a média das distâncias entre todos os pares de valores de um grupo com o outro. Foram processadas duas análises de agrupamento hierárquica: a primeira envolveu 28 áreas, processada com os indices CY_IR, CV_IR e FL_IR; e a segunda, somente 18 áreas, processada com os indices CY_V/m, CV_V/m e FL_V/m, excluídos aqueles grupos com valores inexistentes (Tabela 3).

\section{RESULTADOS E DISCUSSÃO}

A primeira análise de agrupamento hierárquica, realizada com os valores de importância relativa, CY_IR, CV_IR e FL_IR, resultou numa arquitetura envolvendo inicialmente dois grandes grupos de talhões, subdivididos em $X$, $Y, Z$ e $W$ (Figura 2). Dentro desses quatro grupos verificou-se haver informações importantes, que podem ser úteis na tomada de decisões quanto às estratégias de controle e manejo. Os cinco talhões que compuseram o grupo $X$ (FM-18, USM-29, FBV-24, FM-15 e USB-11) apresentaram elevada participação de plantas de folha larga propagadas por sementes (FL_IR), baixa participação de Cyperus rotundus (CY_IR) e participações variáveis das cordas-de-viola
(CV_IR), mas que nunca superaram a participação do conjunto das demais plantas de folha larga (FL_IR). Foram identificados dois subgrupos: $X a$ (USB-11), caracterizado pela alta participação de gramíneas (GR_IR), conforme Tabela 3, que ficou fora da análise; e $X b$ (FM15, FM-18, FBV-24 e USM-29), caracterizado pela baixa participação dos demais conjuntos. Durante vários anos o foco principal das indústrias de herbicidas presentes no mercado de cana-de-açúcar e dos canavicultores foram plantas daninhas das familias Graminae (Poaceae) e Cyperaceae, pois nessas familias sempre se destacavam algumas espécies entre as principais e que causavam os maiores danos. Os herbicidas especificos para as dicotiledôneas, como o 2,4-D, tiveram sua utilização bastante reduzida, porém as sementes de algumas destas espécies, por apresentarem dormência e longevidade, permaneceram no solo e, com a mudança no sistema, vêm aumentando de importância nos canaviais.

O grupo $Y$ foi composto por sete talhões (FSO-26, FSI-8, FBV-22, UDP-6, FBV-23, FBV25 e FBV-5), que se caracterizaram pela maior participação das cordas-de-viola (CV_IR) e participações variáveis de $C$. rotundus (CY_IR) e folhas largas (FL_IR), mas que nunca superaram a participação das cordas-de-viola (Tabela 3). Dependendo da participação do conjunto composto pelas folhas largas (FL_IR), puderam ser distinguidos três subgrupos: $Y a$ (FBV-5, FBV-25, FBV-23 e UDP-6), caracterizado pela baixa participação de folhas largas (FL_IR); Yb (FBV-22), caracterizado por participação significativa das plantas de folha larga (FL_IR); e Yc (FSI-8 e FSO-26), caracterizado pelo equilíbrio na participação das cordas-deviola (CV_IR) e das demais plantas de folha larga (FL_IR). Um fator importante e que pode ter contribuído para o estabelecimento desse padrão de comunidade é que a germinação e a emergência de várias espécies de corda-deviola são pouco afetadas pela camada de palha (Martins et al., 1999; Azania et al., 2002; Gravena et al., 2004). Além disso, as colhedeiras introduzidas neste sistema funcionam como eficientes agentes de disseminação, fazendo com que as populações se espalhem rapidamente nos diferentes talhões.

Os talhões que se encaixaram no grupo $Z$ (FSC-4, FM-16, UAN-7, FM-14, COP-13, FSI-9, 
Tabela 3 - Valores de importância relativa (IR) e de índice de agregação (V/m) para os diferentes conjuntos de plantas utilizados como variáveis nas análises de agrupamento

\begin{tabular}{|c|c|c|c|c|c|c|}
\hline Área & CY_IR & CV_IR & FL_IR & CY_V/m & $\mathrm{CV} \_\mathrm{V} / \mathrm{m}$ & FL_V/m \\
\hline FSI-1 & 22,60 & 0,00 & 0,00 & 18,59 & 38,68 & 1,33 \\
\hline FBV-2 & 99,78 & 0,03 & 0,19 & 69,20 & 8,83 & 1,97 \\
\hline NSF-3 & 100,00 & 0,00 & 0,00 & 45,07 & 9,00 & 1,05 \\
\hline FSC-4 & 83,15 & 0,06 & 16,74 & 1807,62 & 20,32 & 226,52 \\
\hline FBV -5 & 0,60 & 99,33 & 0,00 & 22,89 & 27,07 & 6,56 \\
\hline UDP-6 & 0,00 & 97,26 & 2,70 & \# & 20,48 & 5,43 \\
\hline UAN-7 & 95,94 & 0,00 & 3,82 & 48,00 & 1,00 & 22,83 \\
\hline FSI-8 & 1,85 & 39,30 & 58,86 & 15,00 & 14,27 & 28,50 \\
\hline FSI-9 & 99,92 & 0,01 & 0,00 & 727,07 & 16,37 & 5,13 \\
\hline USB-10 & 100,00 & 0,00 & 0,00 & 34,96 & $\#$ & $\#$ \\
\hline USB-11 & 0,00 & 2,06 & 75,31 & $\#$ & 16,38 & 129,35 \\
\hline COP-13 & 99,20 & 0,00 & 0,58 & 270,68 & $\#$ & 6,97 \\
\hline FM-14 & 99,22 & 0,31 & 0,46 & 47,42 & 1,52 & 13,69 \\
\hline FM-15 & 0,45 & 1,88 & 97,67 & 2,00 & 2,00 & 4,13 \\
\hline FM -16 & 91,51 & 6,52 & 1,97 & 23,87 & 1,78 & 3,04 \\
\hline FSA-17 & 1,49 & 3,13 & 0,29 & 1,86 & 1,40 & 0,80 \\
\hline FM - 18 & 8,88 & 0,12 & 91,00 & 11,12 & 1,93 & 3,34 \\
\hline USM-19 & 100,00 & 0,00 & 0,00 & 71,08 & 1,52 & 1,78 \\
\hline USM-20 & 100,00 & 0,00 & 0,00 & 60,64 & 1,00 & 1,78 \\
\hline FSC-21 & 99,96 & 0,03 & 0,00 & 195,00 & 3,22 & 3,12 \\
\hline FBV-22 & 0,00 & 79,52 & 19,11 & $\#$ & 6,10 & 5,67 \\
\hline FBV-23 & 1,32 & 97,47 & 0,73 & 14,00 & 6,18 & 2,33 \\
\hline FBV-24 & 0,00 & 0,00 & 100,00 & $\#$ & $\#$ & 2,29 \\
\hline FBV-25 & 0,00 & 98,82 & 1,18 & $\#$ & 1,00 & 0,78 \\
\hline FSO-26 & 0,00 & 58,17 & 41,66 & $\#$ & 1,84 & 2,25 \\
\hline FSO-27 & 46,88 & 2,17 & 10,43 & 53,00 & 1,22 & 1,10 \\
\hline USM-28 & 46,58 & 0,00 & 53,42 & 29,49 & $\#$ & 1,96 \\
\hline USM-29 & 0,00 & 0,00 & 100,00 & $\#$ & $\#$ & 3,22 \\
\hline Média & 46,39 & 20,94 & 24,15 & 226,58 & 9,10 & 18,70 \\
\hline
\end{tabular}

\# Valor inexistente.

FSC-21, USM-20, USM-19, USB-10, NSF-3 e FBV-2) caracterizaram-se pela alta participação de $C$. rotundus, com elevados valores de CY_IR e baixa participação dos outros conjuntos de espécies e baixos valores de CV_IR e FL_IR. Esse grupo acolheu 12 talhões estudados e apresentou talhões com elevada similaridade, com distâncias euclidianas muito próximas de zero (FBV-2, NSF-3, USB-10, USM19, USM-20, FSC-21, FSI-9, COP-13 e FM-14) e talhões com maior diferenciação, ou seja, maiores distâncias (FSC-4, FM-16 e UAN-7), levando à formação de dois subgrupos distintos: $Z a$ e $Z b$. Os dois subgrupos apresentaram em comum a elevada participação de $C$. rotundus
(CY_IR), acima de 90\%; entretanto, o subgrupo $Z b$ apresentou baixa participação, porém significativa, das cordas-de-viola (CV_IR) (FM-16) ou plantas de folha larga propagadas por sementes (FL_IR) (FSC-4 e UAN 7). Das espécies importantes no sistema tradicional de colheita, ou seja, manual queimada, Cyperus rotundus foi uma das que mantiveram a sua participação nos canaviais com grande importância, confirmando resultados de pesquisas que apontavam essa tendência (Silva et al., 2003; Durigan et al., 2006).

Os talhões que se encaixaram no grupo $W$ (FSI-1, FSC-17, FSO-27 e USM-28) tiveram 
participação de C. rotundus, com CY_IR variando entre 1,49 e 46,88\%. Em função da participação diferencial das demais espécies, constatou-se que houve divisão em dois subgrupos: Wa, cujos talhões apresentaram grande participação de variáveis que ficaram ausentes da análise de agrupamento (GR_IR e PER_IR), conforme pode ser observado na Tabela 3; e Wb (USM-28), caracterizado por equilíbrio entre a participação das plantas de folha larga e tiririca (CY_IR e FL_IR). A presença de plantas perenes, entre elas Vernonia polyanthes, Cissampelos glaberrima, Pyrostegia venusta, Talinum paniculatum e Mikania cordifolia, antes restritas às áreas de pastagens ou áreas marginais, pode estar relacionada à redução na freqüência de distúrbio mecânico no solo. Além disso, essas plantas, quando ceifadas pela colhedeira, podem estar sendo induzidas a desenvolver mais intensamente o sistema subterrâneo, dificultando o controle pelos herbicidas registrados para a cana-deaçúcar, que são naturalmente pouco eficientes para essas plantas. A ocorrência destas plantas indica que os herbicidas hoje utilizados em pastagens podem ser futuramente aproveitados na cultura da cana-de-açúcar para conter o avanço dessas populações.
A segunda análise de agrupamento hierárquica, realizada com os índices de agregação, $\mathrm{CY}$ _V / m, CV_V/m e FL_V/m, resultou numa arquitetura de grupos envolvendo inicialmente dois grupos de talhões - R e S (Figura 3), ficando três talhões isolados (FSC-4, FSI-9 e FSC 21). Dentro desses dois grupos verificouse haver informações importantes, que podem ser úteis na tomada de decisões quanto às estratégias de controle e manejo.

O talhão FSC-4 apresentou forte agregação para Cyperus rotundus $(\mathrm{CY}$-V/m $=1807,62)$, para as cordas-de-viola $\left(\mathrm{CV}_{-} \mathrm{V} / \mathrm{m}=20,32\right)$ e para as plantas de folha larga propagadas por sementes $\left(F L \_V / m=226,52\right)$. O talhão FSI-9 apresentou forte agregação de Cyperus rotundus (CY_V/m $=727,07)$ e para as cordas-de-viola $(\mathrm{CV}$ _V $/ \mathrm{m}=16,37)$, e o talhão FSC 21 , somente para Cyperus rotundus $\left(\mathrm{CY}_{-} \mathrm{V} / \mathrm{m}=195\right)$.

O grupo $R$ caracterizou-se por apresentar padrão agregado de distribuição com valores médios, dentro da amplitude dos dados, para C. rotundus (CY_V/m). Os indices variaram entre 45 e 71. Em função dos indices de agregação dos outros dois conjuntos de espécies, puderam ser separados em três subgrupos: $R a, R b$ e $R c$. O subgrupo $R a$, formado pelos

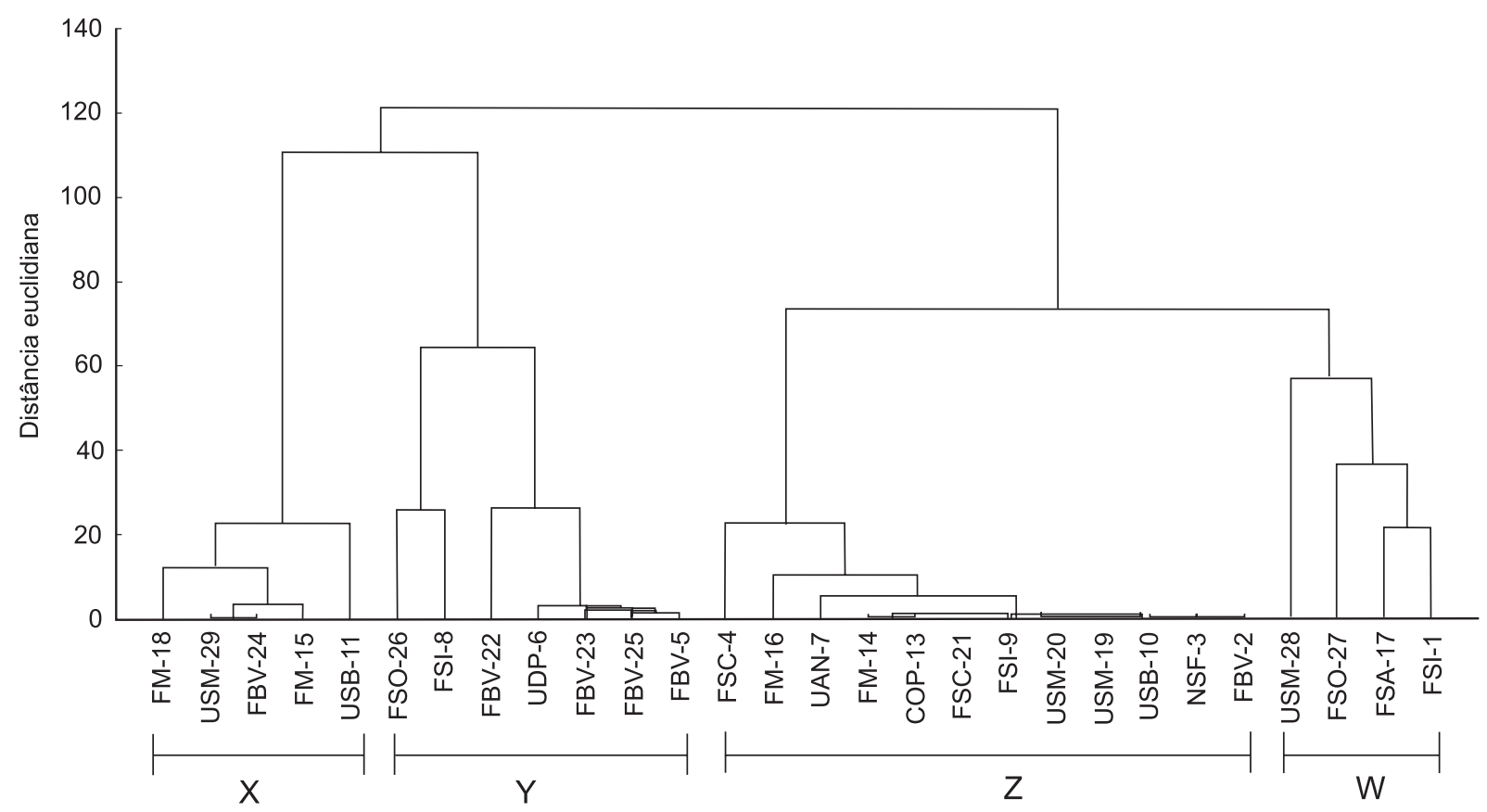

Figura 2 - Dendrograma resultante da análise de agrupamento hierárquica, utilizando a importância relativa (IR) dos diferentes conjuntos de plantas daninhas, calculado a partir de dados coletados aos 120 dias após o corte da cana. 
talhões FSO-27, USM-20 e NSF-3, mostrou baixos valores de agregação para as cordasde-viola $\left(\mathrm{CV}_{-} \mathrm{V} / \mathrm{m}\right)$ e para as folhas largas (FL_V / m); $R b$, formado pelos talhões FBV-2 e USM-19, apresentou baixos valores de agregação para folhas largas (FL) e médio para as cordas-de-viola $(\mathrm{CV}$ _V/m), ao passo que $R c$, representado pelos talhões UAN-7 e FM-14, apresentou baixa agregação para as cordas-de-viola $\left(\mathrm{CV}_{-} \mathrm{V} / \mathrm{m}\right)$ e média para as plantas de folha larga $\left(\mathrm{FL} \_\mathrm{V} / \mathrm{m}\right)$.

O grupo $S$ caracterizou-se por baixos valores de agregação para Cyperus rotundus (CY_V/ $\mathrm{m}<30$ ). Em uma análise mais detalhada dentro do grupo, avaliando os demais conjuntos de plantas, puderam ser identificados dois subgrupos. O subgrupo $\mathrm{Sa}$ foi formado pelos talhões FM-15, FM-16, FM-18, FSA-17 e FBV-23, que apresentaram em comum os mais baixos valores de agregação para todos os conjuntos considerados, aproximando-se em várias ocasiões do padrão aleatório de distribuição $(\mathrm{V} / \mathrm{m}=1)$, principalmente para as cordas-de-viola $\left(\mathrm{CV}_{-} \mathrm{V} /\right.$ $\mathrm{m})$ e demais plantas de folha larga (FL_V/m). O subgrupo $S b$ foi composto pelos talhões FSI1, FBV-5 e FSI-8, que mostraram valores de agregação elevados para as cordas-de-viola.
A tiririca é uma planta de propagação vegetativa e sua disseminação depende muito das atividades realizadas pelo homem. Na canade-açúcar colhida mecanicamente, ocorre redução da movimentação do solo por meio dos cultivadores, contribuindo para a redução de sua disseminação. A camada de palha por si só é incapaz de conter a emergência e o desenvolvimento da tiririca (Durigan al., 2006), podendo levar à formação de populações extremamente agregadas, conforme pode ser observado nos talhões FSC-4, FSI-9 e FSC-1. Em contrapartida, plantas propagadas por sementes, sobretudo aquelas que possuem tamanho relativamente grande, com quantidade de reservas suficiente para garantir a passagem da camada de palha, podem estar sendo beneficiadas. As colhedeiras de cana-de-açúcar funcionam como excelentes agentes de disseminação de plantas daninhas, especialmente quando a época de colheita da cana coincide com o fim do ciclo de seu desenvolvimento e maturação de suas sementes. As principais espécies de corda-de-viola, em algumas ocasiões, apresentam essa sincronia e, nesse caso, ao longo do tempo tendem a formar grandes populações, porém com menores índices de agregação.

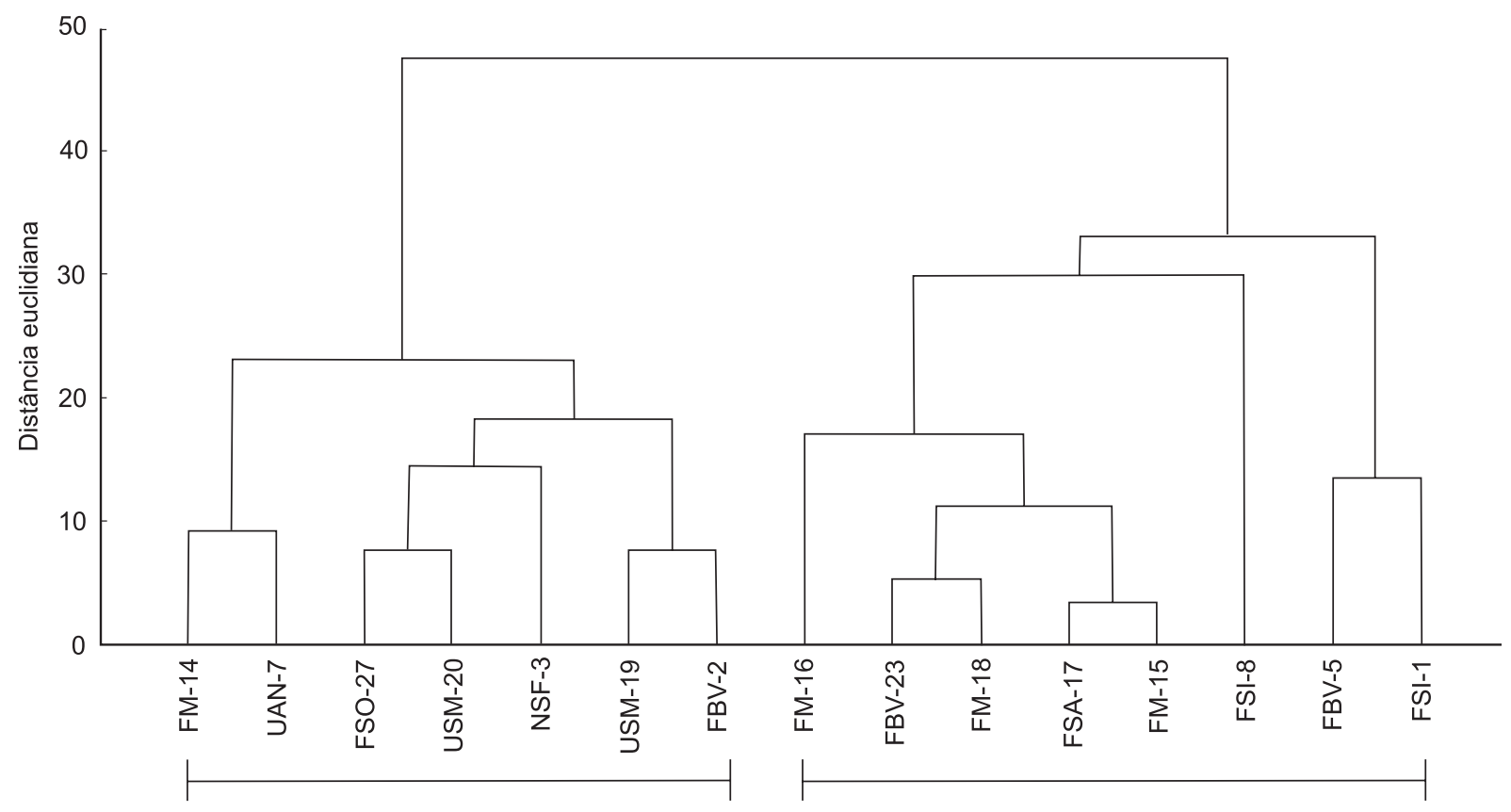

Figura 3 - Dendrograma resultante da análise de agrupamento hierárquica, utilizando o índice de agregação (V/m) dos diferentes conjuntos de plantas daninhas, calculado a partir de dados coletados aos 120 dias após o corte da cana. 
Alguns trabalhos, entre os quais os de Shiratsuchi (2001), Green \& Vencil (1997), Engqvist et al. (1997) e Heisel et al. (1996), destacaram o padrão agregado de populações de plantas daninhas e obtiveram economia de herbicida com a aplicação diferencial. Essa diferenciação nas taxas de aplicação dentro de um mesmo talhão ainda é de difícil adoção em escala comercial, pois envolve o desenvolvimento de outras tecnologias, como a obtenção de mapas de distribuição e sensores. Entretanto, é possivel prever, com os resultados obtidos, que a simples diferenciação entre talhões ou mesmo glebas com base no conhecimento da sua composição de plantas daninhas, distribuição e organização em padrões poderá acarretar economia de despesas de controle.

Pesquisas vêm sendo desenvolvidas buscando metodologias capazes de diferenciar de forma rápida e eficiente plantas daninhas, plantas cultivadas e solo descoberto com utilização de imagens e técnicas de análises multivariadas (Permonian, 2002; Okamoto et al., 2007). Ao mesmo tempo, metodologias para geração de mapas de distribuição de plantas daninhas nas lavouras num curto espaço de tempo estão sendo pesquisadas (Ye et al., 2007). Com o domínio dessas técnicas, outro passo importante para o manejo de plantas daninhas será identificar a proporção das espécies numa comunidade de plantas daninhas e a obtenção de padrões de infestação.

Nos dois dendrogramas obtidos não foi observada nenhuma relação entre os grupos formados e a época de corte dos talhões. A análise de agrupamento padroniza os talhões formando grupos, mas associar os grupos com práticas culturais adotadas e suas implicações (quantidade de palha, época de corte, espaçamento, adubação, etc.) não é uma tarefa simples e requer um banco de dados com maior quantidade de informações. Além disso, as variáveis associadas às práticas culturais, como, por exemplo, a época de corte, contêm embutidas em si outras inúmeras variáveis ambientais, entre elas temperatura, precipitação pluvial, insolação e umidade relativa.

Com este trabalho, concluiu-se que foi possivel estabelecer padrões de comunidades de plantas daninhas do agroecossistema canacrua por meio de técnicas estatísticas multivariadas de agrupamento hierárquico. Utilizando variáveis do tipo importância relativa (IR), foi possivel identificar quatro grupos de padrão de infestação. Por outro lado, com variáveis do tipo índice de agregação (V/ $\mathrm{m})$, foi possivel identificar dois grupos de padrão de infestação, bem como três talhões isolados com padrões específicos.

\section{AGRADECIMENTOS}

À Prof ${ }^{a}$ Drª Maria do Carmo Morelli Damasceno Pavani, pelo auxílio na identificação das plantas daninhas; à Dow Agrosciences Ltda., Milenia Agro Ciências Ltda e aos agrônomos e técnicos das usinas e fazendas envolvidas (U. Bonfim, U. da Pedra, U. Andrade, F. Santa Isabel, F. Santa Cecília, F. Santo Antonio), pelas diversas formas de apoio.

\section{LITERATURA CITADA}

FNP CONSULTORIA \& COMÉRCIO. Agrianual 2006: Anuário da agricultura brasileira. São Paulo: 2006. p. 227247.

AZANIA, A. A. P. M. et al. Interferência da palha de canade-açúcar (Saccharum spp.) na emergência de espécies de plantas daninhas da família Convolvulaceae. Planta Daninha, v. 20, n. 2, p. 207-212, 2002.

DURIGAN, J. C.; TIMOSSI, P. C.; CORREIA, N. M. Manejo integrado da tiririca na produtividade da cana-deaçúcar. Planta Daninha, v. 24, n. 1, p.77-81, 2006.

ENGQVIST, A.; BENGTSSON, P.; ENFALT, P. A model for site-specific broad-leaved weed control based on weed plants variables. In: THE EUROPEAN CONFERENCE ON PRECISION AGRICULTURE, 1., 1997, Warwick.

Proceedings... Oxford: BIOS Scientific, 1997. p. 869-878.

GRAVENA, R. et al. Controle de plantas daninhas através da palha de cana-de-açúcar associada à mistura dos herbicidas trifloxysulfuron sodium + ametrina. Planta Daninha, v. 22, n. 2 , p. $419-427,2004$

GREEN, H. M.; VENCIL, W. K. Precision management of spatially variable weeds. In: THE EUROPEAN CONFERENCE ON PRECISION AGRICULTURE, 1 , 1987, Warwick, Proceedings... Oxford: BIOS Scientific, 1997. p. 983-989.

HAIR, J. F. et al. Análise multivariada de dados. 5.ed. Porto Alegre: Bookman, 2005. 593 p. 
HEISEL, T.; ANDREASEN, C.; ERSBOLL, A. K. Annual weed distributions can be mapped with krigging. Weed Res., v. 36, p. 325-337, 1996.

KUVA, M. A. et al. Fitossociologia de comunidades de plantas daninhas em agroecossistema cana-crua. Planta Daninha, v. 25, n. 3, p. 501-511, 2007.

LARA, J. F. R.; MACEDO, J. F.; BRANDÃO, M. Plantas daninhas em pastagens de várzeas no Estado de Minas Gerais. Planta Daninha, v. 21, n. 1, p. 11-20, 2003.

MACEDO, J. F.; BRANDÃO, M.; LARA, J. F. R. Plantas daninhas na pós-colheita de milho nas várzeas do rio São Francisco, em Minas Gerais. Planta Daninha, v. 21, n. 2, p. 239-248, 2003.

MARTINS, D. et al. Emergência em campo de dicotiledôneas infestantes em solo coberto com palha de cana-de-açúcar. Planta Daninha, v. 17, n. 1, p. 151-161, 1999

MUELLER-DOMBOIS, D.; ELLEMBERG, H. Aims and methods of vegetation ecology. New York: John Wiley \& Sons, 1974. 547 p.

ODUM, E. P. Ecologia. Rio de Janeiro: Interamericana, 1985. $434 \mathrm{p}$.

OKAMOTO, H. et al. Plant classification for weed detection using hyperspectral imaging with wavelet analusis. Weed Biol. Manag., v. 7, n. 1, p. 31-37, 2007.

PERMONIAN, V. A. Identificação de plantas invasoras em tempo real. 2002. 113 f. Dissertação (Mestrado em Ciências da Computação e Matemática Computacional) Universidade de São Paulo, São Carlos, 2002.
PITELLI, R. A. Inteferência de plantas daninhas em culturas agrícolas. Inf. Agropec., v. 11, n. 129, p. 16-27, 1985.

ROWE, M. L. et al. Genetic variation in North America leafy spurge (Euphorbia esula) determined by DNA markers. Weed Sci., v. 45, n. 3, p. 446-454, 1997.

SHIRATSUCHI, L. S. Mapeamento da variabilidade espacial das plantas daninhas com utilização de ferramentas da agricultura de precisão. 2001. $96 \mathrm{f}$. Dissertação (Mestrado em Fitotecnia) - Escola Superior de Agricultura Luiz de Queiroz, Piracicaba, 2001.

SILVA, J. R. V.; COSTA, N. V.; MARTINS, D. Efeito da palhada de cultivares de cana-de-açúcar na emergência de Cyperus rotundus. Planta Daninha, v. 21, n. 3, p. 373-380, 2003.

SOUZA, L. S. A.; SILVA, J. F.; SOUZA, M. D. B. Composição florística de plantas daninhas em agrossistemas de cupuaçuzeiro (Theobroma grandiflorum) e pupunheira (Bactris gasipaes). Planta Daninha, v. 21, n. 2, p. 249-255, 2003.

WINKLER, L. M.; VIDAL, R. A.; BARBOSA NETO, J. F. Caracterização genética de Euphorbia heterophylla resistente a herbicidas inibidores de acetolactato sintase. Pesq. Agropec. Bras., v. 38, n. 9, p. 1067-1072, 2003.

YE, X. et al. Use of airborne multispectral imagery to discriminate and map weed infestation in a citrus orchard. Weed Biol. Manag., v. 7, n. 1, p. 23-30, 2007. 